\title{
Germinação de sementes de sempre-vivas (Syngonanthus elegans e S. venustus)
}

\author{
FERNANDACRISTIANE SIMÕES(1), PATRÍCIA DUARTE DE OLIVEIRA PAIVA(2), \\ MARIALAENE MOREIRADE CARVALHO(2) THAÍSA SILVATAVARES(3), eRENATO PAIVA ${ }^{(4)}$
}

\begin{abstract}
RESUMO
A sempre-viva é uma espécie ornamental nativa, com ocorrência endêmica em algumas regiões brasileiras. Atualmente, encontra-se entre as principais espécies exportadas e seu consumo interno também é expressivo. Mas, apesar da sua importância comercial, poucas são as informações agronômicas sobre seu cultivo. Sabe-se que são propagadas por meio de sementes, preparadas pelos próprios produtores de flores. Assim, visando avaliar características das sementes, coletaram-se capítulos florais de Syngonanthus elegans e $S$. venustus - cultivados na região de Diamantina (MG) - dos quais se retiraram as sementes, que foram submetidas a testes de germinação, avaliando-se efeitos das temperaturas de 20, 25 , 30 e $35^{\circ} \mathrm{C}$. Utilizaram-se quatro repetições com 50 sementes em cada caixa plástica tipo gerbox. As sementes das duas espécies tiveram maior percentagem e velocidade de germinação a $25^{\circ} \mathrm{C}$, mas não germinaram quando submetidas à temperatura de $35^{\circ} \mathrm{C}$. As sementes de Syngonanthus venustus também tiveram primeira contagem e IVG (Índice de velocidade de germinação) superiores às de Syngonanthus elegans.

Palavras-chave: Eriocaulaceae, floricultura, flores secas.
\end{abstract}

\section{ABSTRACT \\ Seed germination of dried flowers Syngonanthus olegan e $S$. venustus}

Syngonanthus sp. is a native ornamental plant endemic to Brazilian savannahs. It has been considered one of the most important exported species and also presents an expressive inner consumption. Although it presents a commercial importance little is known of its agronomic characteristics. It is propagated by seeds prepared by the producers. With the objective to evaluate seed characteristics, flower chapels of $S$. elegans and $S$. venustus were collected and the seeds were extracted and submitted to germination by evaluating the effect of temperature $\left(20,25,30\right.$ and $\left.35{ }^{\circ} \mathrm{C}\right)$. Four replicates with 50 seeds in each gerbox type plastic tray were used. The seeds of both species presented higher percentage and germination velocity at $25{ }^{\circ} \mathrm{C}$ with no germination being observed at $35^{\circ} \mathrm{C}$. The seeds of $S$. venustus showed the first count and germination speed index superior than seeds of $S$. elegans.

Key words: Eriocaulaceae, flower culture, dry flowers

\section{INTRODUÇÃO}

As espécies de eriocauláceas, conhecidas popularmente como sempre-vivas (TEIXEIRA 1987; GIULIETTI et al. 1987; GIULIETTI et al. 1988), constituemse de diversas plantas nativas das serras e cerrados, que ocorrem especialmente ao longo da Cadeia do Espinhaço (MOLDENKE E SMITH, 1976, NÉRI et al. 2005). Os gêneros Syngonanthus, Paepalanthus e Eriocaulon estão entre os que apresentam maior número de espécies (cerca de 270, 400 e 400 espécies, respectivamente).

Algumas espécies são largamente usadas para fins de decoração, sendo também produto de exportação do Brasil. Esse fato confere a essas plantas alto valor comercial, principalmente no mercado internacional e seu extrativismo constitui-se importante atividade econômica nas suas regiões de ocorrência (GIULIETTI et al., 1987; NERI et al., 2005).

A espécie Syngonanthus elegans (Bong.) Ruhl., conhecida popularmente como sempre-viva pé-de-ouro, é uma das espécies mais exploradas comercialmente por possuir o escapo longo. A espécie Syngonanthus venustus Silveira, conhecida como pé-liso, por não possuir pilosidade em sua base (o que causa alergia em algumas pessoas), é também bastante explorada (NERI et al., 2005). A propagação dessas espécies é feita por meio de sementes, extraídas de plantas destinadas à comercialização de flores, não sendo feito nenhum processo de seleção ou tratamento. Os produtores simplesmente extraem as sementes e realizam a semeadura a lanço nas áreas de produção. Não há registros também sobre características de germinação dessas espécies.

O conhecimento de características germinativas das sementes desse grupo de plantas é bastante limitado e os poucos trabalhos disponíveis analisaram aspectos morfológicos e uso de reguladores de crescimento, mas não aspectos germinativos.

Devido à importância socioeconômica e o potencial ornamental do gênero Syngonanthus, bem como sua vulnerabilidade frente à coleta predatória, este trabalho

\footnotetext{
(1)Engenheira Agrônoma, Dra. Campinas (SP).

(2) Professora do Departamento de Agricultura, Universidade Federal de Lavras - UFLA - Lavras (MG ).

${ }^{(3)}$ Engenheira Agrônoma pela Universidade Federal de Lavras - UFLA- Lavras (MG).

${ }^{(4)}$ Professor do Departamento de Biologia, Universidade Federal de Lavras - UFLA - Lavras (MG).
} 
foi realizado objetivando avaliar a influência da temperatura sobre o comportamento germinativo de Syngonanthus elegans (Bong.) Ruhl. e S. venustus Silveira.

\section{MATERIALE MÉTODOS}

O presente trabalho foi realizado no Laboratório de Análises de Sementes do Departamento de Agricultura da Universidade Federal de Lavras (MG) onde foram conduzidos testes para avaliar a porcentagem de germinação das sementes.

As sementes de sempre-vivas foram obtidas de flores dispostas em galpão de armazenamento de um produtor de sempre-vivas da cidade de Diamantina (MG). Utilizaram-se para o experimento duas espécies diferentes: Syngonanthus elegans e $S$. venustus, das quais se retiraram sementes dos capítulos florais após um período de, aproximadamente, sete meses de armazenamento. Após a retirada dos capítulos, as sementes foram passadas por peneira fina para retirar impurezas.

No laboratório, o teste de germinação foi conduzido colocando-se as sementes de cada espécie em caixas de plástico tipo gerbox $(11,0 \times 11,0 \times 3,5 \mathrm{~cm})$, sobre duas folhas de papel (tipo mata-borrão), umedecidas com quantidade de água equivalente a 2,5 vezes o peso do papel do substrato. As caixas de plástico com as sementes foram mantidas em câmaras tipo BOD, reguladas com temperaturas de $20,25,30$ e $35^{\circ} \mathrm{C}$.

Os resultados foram expressos em porcentagem média de plântulas germinadas para cada espécie. O delineamento experimental utilizado foi o inteiramente casualizado com quatro tratamentos (temperaturas) e quatro repetições de 50 sementes cada. Foi realizado um teste para cada espécie, nas mesmas condições.

As avaliações foram realizadas aos 23 dias após a instalação do teste, seguindo-se os critérios estabelecidos pelas Regras para Análises de Sementes (BRASIL, 1992), obtendo-se a porcentagem média de germinação para tratamento. Também foram obtidos os dados da primeira contagem, da velocidade e da percentagem final de germinação das sementes.
As avaliações foram realizadas diariamente, à mesma hora, a partir do dia em que surgiram as primeiras sementes germinadas. Esse procedimento prosseguiu até o dia da última contagem.

Ao final do teste, com os dados diários do número de plântulas normais, calculou-se a velocidade de germinação empregando a fórmula de MAGUIRE (1962):

$$
\text { IVG: } \frac{G 1}{N 1}+\frac{G 2}{N 2}+\ldots+\frac{G n}{N n}
$$

Onde: IVG: índice de velocidade de germinação;

$\mathrm{G}_{1}, \mathrm{G}_{2}, \mathrm{G}_{\mathrm{n}}$ : número de plântulas contadas;

$\mathrm{N}_{1}, \mathrm{~N}_{2}, \mathrm{~N}_{\mathrm{n}}$ : número de dias a partir da semeadura.

A velocidade de germinação e, conseqüentemente, o vigor das sementes foram proporcionais aos valores obtidos (MAGUIRE, 1962).

Para análise estatística, os dados de germinação de $S$. elegans, expressos em percentagem, foram transformados em arco seno da raiz quadrada da percentagem e submetidos à análise de variância (ANOVA). As comparações entre as médias foram feitas mediante a aplicação do teste de Tukey ao nível de 5\% de probabilidade.

\section{RESULTADOS E DISCUSSÃO}

Na tabela 1, encontram-se os dados referentes aos testes de germinação (\%), sementes mortas (\%), sementes dormentes (\%), índice de velocidade de germinação (IVG) e primeira contagem para a análise de germinação da espécie $S$. elegans. Os resultados para $S$. venustus estão relacionados na tabela 2 .

As sementes de Syngonanthus elegans e $S$. venustus germinaram quando mantidas a $25{ }^{\circ} \mathrm{C}$, não ocorrendo esse processo quando expostas à temperatura de $35^{\circ} \mathrm{C}$ (tabela 1). Comparando-se o comportamento das duas espécies nessa de temperatura, a germinação de $S$. venustus (62\%) foi superior à de S.elegans (25\%). Em todas as outras características avaliadas, a espécie $S$. venustus também obteve valores maiores em relação à $S$. elegans.

Tabela 1. Germinação (\%), sementes mortas (\%), sementes dormentes (\%), índice de velocidade de germinação (IVG) e primeira contagem em sementes de sempre-viva pé-de-ouro (Syngonanthus elegans).

Germination (\%), death seeds (\%), dormant seeds (\%), germination velocity index (GVI) and first count of Syngonanthus elegans seeds

\begin{tabular}{cccccc}
\hline $\begin{array}{c}\mathrm{T} \\
\left({ }^{\circ} \mathrm{C}\right)\end{array}$ & $\begin{array}{c}\text { Germinação } \\
(\%)\end{array}$ & $\begin{array}{c}\text { Sementes mortas } \\
(\%)\end{array}$ & $\begin{array}{c}\text { Sementes dormentes } \\
(\%)\end{array}$ & IVG & $\begin{array}{c}\text { Primeira } \\
\text { contagem }\end{array}$ \\
\hline 20 & $18,0 \mathrm{a}$ & $22,0 \mathrm{a}$ & $61,0 \mathrm{a}$ & $7,0 \mathrm{~b}$ & $0 \mathrm{~b}$ \\
25 & $25,0 \mathrm{a}$ & $13,0 \mathrm{~b}$ & $62,0 \mathrm{a}$ & $19,0 \mathrm{a}$ & $3,0 \mathrm{~b}$ \\
30 & $19,0 \mathrm{a}$ & $13,0 \mathrm{~b}$ & $68,0 \mathrm{a}$ & $20,0 \mathrm{a}$ & $7,0 \mathrm{a}$ \\
35 & $0 \mathrm{~b}$ & $30,0 \mathrm{a}$ & $71,0 \mathrm{a}$ & $0 \mathrm{c}$ & $0 \mathrm{~b}$ \\
\hline
\end{tabular}

Médias seguidas de mesma letra na coluna, não diferem entre si, pelo teste de Tukey a 5\% de probabilidade. 
Em relação à presença de sementes mortas não se observou diferença quando as sementes foram mantidas nas temperaturas de 20 e $35{ }^{\circ} \mathrm{C}$, sendo a ocorrência superior, em comparação com as sementes mantidas a 25 e $30^{\circ} \mathrm{C}$. Efeito semelhante foi observado para as sementes da espécie $S$. venustus (tabela 2).

Tabela 2. Germinação (\%), sementes mortas (\%), sementes dormentes (\%), índice de velocidade de germinação(IVG) e primeira contagem em sementes de sempre-viva pé-liso (Syngonanthus venustus)

Germination (\%), death seeds (\%), dormant seeds (\%), germination velocity index (GVI) and first count of Syngonanthus venustus seeds

\begin{tabular}{cccccc}
\hline $\begin{array}{c}\mathrm{T} \\
\left({ }^{\circ} \mathrm{C}\right)\end{array}$ & $\begin{array}{c}\text { Germinação } \\
(\%)\end{array}$ & $\begin{array}{c}\text { Sementes mortas } \\
(\%)\end{array}$ & $\begin{array}{c}\text { Sementes dormentes } \\
(\%)\end{array}$ & IVG & $\begin{array}{c}\text { Primeira } \\
\text { contagem }\end{array}$ \\
\hline 20 & $37,0 \mathrm{~b}$ & $48,0 \mathrm{a}$ & $16,0 \mathrm{~b}$ & $14,0 \mathrm{c}$ & $0 \mathrm{~b}$ \\
25 & $62,0 \mathrm{a}$ & $19,0 \mathrm{~b}$ & $20,0 \mathrm{~b}$ & $55,0 \mathrm{a}$ & $6,0 \mathrm{~b}$ \\
30 & $30,0 \mathrm{c}$ & $23,0 \mathrm{~b}$ & $48,0 \mathrm{a}$ & $37,0 \mathrm{~b}$ & $13,0 \mathrm{a}$ \\
35 & $0 \mathrm{~d}$ & $60,0 \mathrm{a}$ & $41,0 \mathrm{~b}$ & $0 \mathrm{~d}$ & $0 \mathrm{~b}$ \\
\hline
\end{tabular}

Médias seguidas de mesma letra na coluna, não diferem entre si, pelo teste de Tukey a 5\% de probabilidade.

O IVG foi semelhante para as sementes mantidas nas temperaturas 25 e $30^{\circ} \mathrm{C}$, que proporcionaram maior velocidade de germinação em relação às outras temperaturas em que as sementes de $\mathrm{S}$. venustus foram mantidas. O IVG foi superior para as sementes mantidas a $30^{\circ} \mathrm{C}$. Na avaliação de primeira contagem, observou-se valor superior para as sementes de ambas as espécies mantidas na temperatura de $30^{\circ} \mathrm{C}$ em comparação aos outros tratamentos.

A germinação obtida em laboratório é um parâmetro nem sempre expresso na população inicial no campo. Por isso, normalmente, deve ser acompanhado de um teste de vigor. Quanto maior o vigor, maior o potencial das sementes em estabelecer mudas no campo (POLLOCK E ROSS, 1972).

De acordo com SCATENA et al. (1996), as sementes de S. elegans obtiveram porcentagem de germinação de $80 \%$, mas deve-se destacar que esses autores utilizaram sementes coletadas diretamente no campo. No experimento realizado neste trabalho, as sementes já tinham sido colhidas há, aproximadamente, sete meses, e isto pode ter diminuído a porcentagem de germinação, além de afetar as outras variáveis avaliadas. Deve-se considerar que essas sementes representam o material geralmente utilizado pelos produtores para plantio e implantação de nova cultura. Apesar de não haver registros, esse valor pode diminuir com o tempo, interferindo na eficácia do plantio realizado pelos produtores.

Observou-se elevada porcentagem de sementes dormentes principalmente para S. elegans, que teve média de $62 \%$, não ocorrendo diferença entre as sementes mantidas em diferentes temperaturas. Para S. venustus essas porcentagens foram menores, sendo observado menor valor (48\%) para as sementes mantidas a $30^{\circ} \mathrm{C}$.

Esse resultado pode ser devido à diferença de maturação entre as duas espécies, já que estas foram coletadas aleatoriamente em galpão de armazenamento (sem controle de temperatura, seguindo as mesmas técnicas utilizadas pelos produtores), ou a características inerentes a cada espécie.
O IVG se mostrou semelhante entre as duas espécies, destacando a temperatura de $30^{\circ} \mathrm{C}$ como mais favorável à rápida germinação das sementes. Para o IVG, segundo MAGUIRE (1962) quanto maior o valor obtido subtende-se maior velocidade de germinação e, conseqüentemente maior vigor. Em função desses resultados, pode-se considerar que as sementes de $\mathrm{S}$. venustus estavam mais vigorosas em relação às de $S$. elegans. A mesma comparação pode ser efetuada para os resultados de primeira contagem de germinação.

A ocorrência de sementes mortas pode ser causada pela presença de fungos associados às sementes (figura 1). A presença de microrganismos, após o ponto de maturidade fisiológica ou durante o enraizamento das sementes, é sempre prejudicial à sanidade das sementes. Elevadas percentagens de sementes infectadas geralmente estão associadas ao decréscimo no poder germinativo e menor desenvolvimento da plântula (YORINORI,1982). Para ROBERTS (1972) fungos associados a sementes podem ser responsáveis, além da transmissão de doenças na parte aérea e radicular da plântula, pelo decréscimo no potencial de desempenho das sementes e morte de plântulas.

A figura 2 apresenta os estádios de desenvolvimento das sementes de sempre-vivas, germinadas sobre papel, em condições de laboratório. Na figura 2a observa-se o desenvolvimento da folha e nas demais, o desenvolvimento da plântula.

Por meio dos resultados obtidos pode-se observar que as duas espécies estudadas têm semelhanças quanto à temperatura exigida para germinação, $25^{\circ} \mathrm{C}$, mas com porcentagens diferentes.

Resultados parecidos foram observados para o IVG e primeira contagem. De modo geral, as porcentagens de germinação da espécie $S$. venustus foram superiores à $S$. elegans. Considerando a deficiência de conhecimentos agronômicos sobre o cultivo e características dessa espécie, os resultados obtidos poderão ser úteis e indicativos para novas pesquisas sobre essas espécies. 

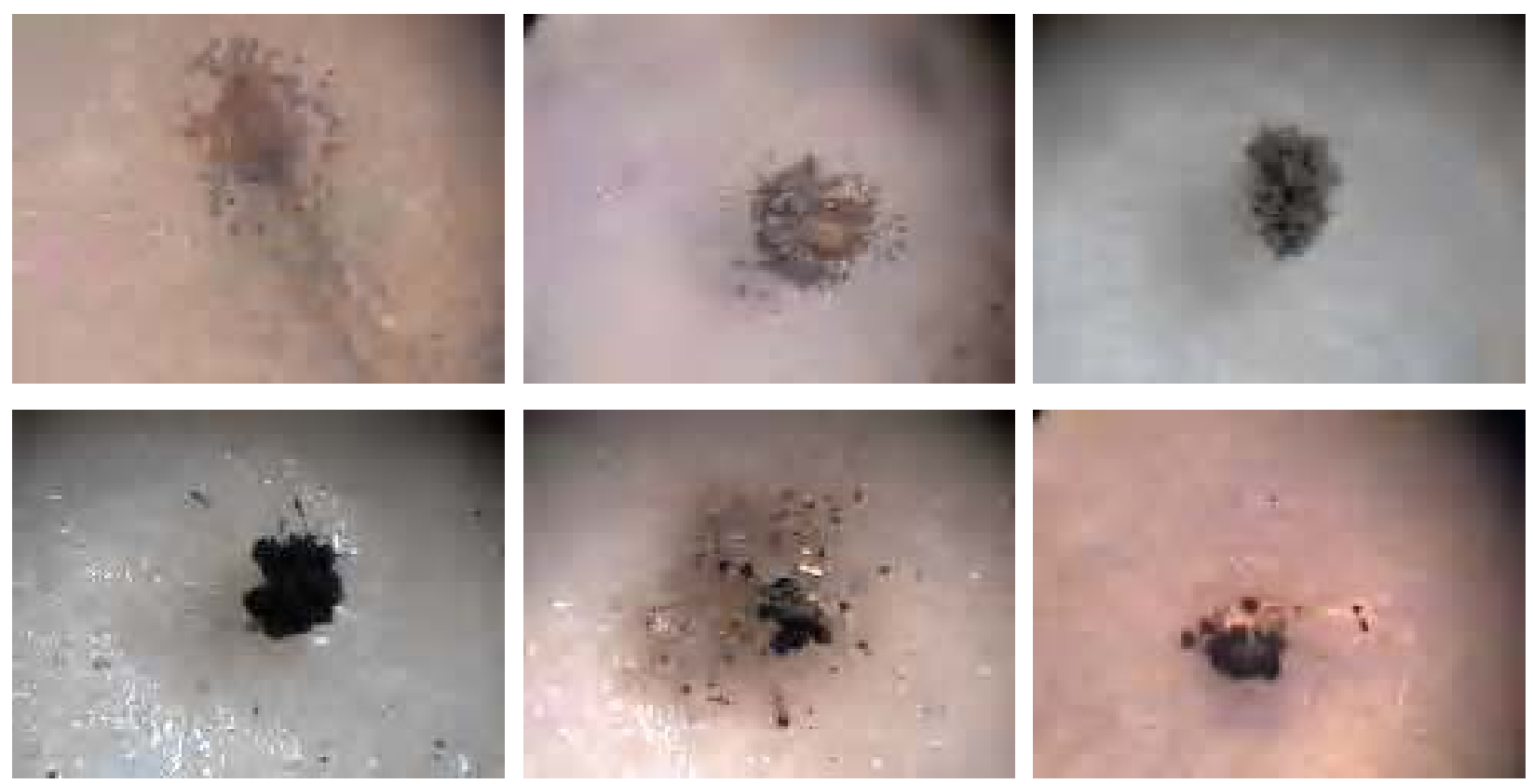

Figura 1. Fungos observados em sementes de sempre-viva (Syngonanthus elegans e S. venustus) durante os testes de análise de sementes.

Fungi observed in Syngonanthus elegans and S. venustus seeds during seed analysis
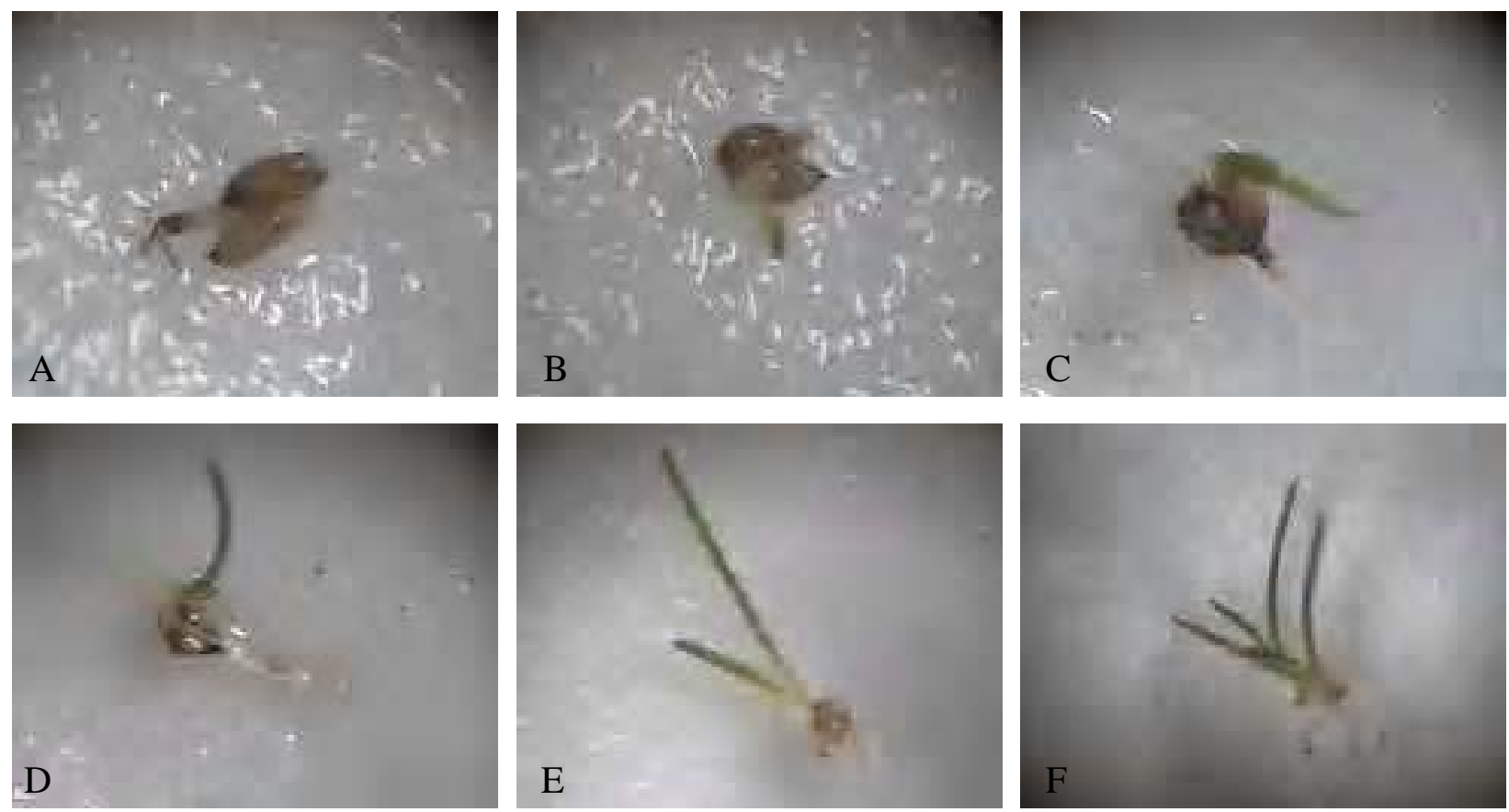

Figura 2. Estádios de desenvolvimento de sementes de sempre-vivas germinadas em condições de laboratório.

(a) desenvolvimento da folha; (b) plântula com 15 dias; (c) plântula com 18 dias; (d) plântula com 21 dias; (e) plântula com 24 dias; (f) plântula com 27 dias.

Developmental stages of Syngonanthus seeds germinated in laboratory conditions. (a) leaf development; (b) 15 days old seedling; (c) 18 days old seedling; (d) 21 days old seedling; (e) 24 days old seedling; (f) 27 days old seedling.

\section{CONCLUSÕES}

Nas condições em que o trabalho foi realizado pode-se concluir que:

- As espécies Syngonanthus elegans e S. venustus obtiveram melhor germinação (62\% e 25\%, respectivamente) na temperatura de $25^{\circ} \mathrm{C}$. Também a velocidade de germinaçãoé maior quando as sementes são expostas a $25^{\circ} \mathrm{C}$.

- Em Syngonanthus elegans os valores de sementes mortas, sementes dormentes e IVG foram superiores aos observados para $S$. venustus.

- Não se observou germinação das sementes mantidas a $35^{\circ} \mathrm{C}$. - A presença de fungos contribuiu para a morte de elevada porcentagem das sementes, de até $60 \%$ a $35^{\circ} \mathrm{C}$. 


\section{REFERÊNCIAS}

BRASIL. Ministério da Agricultura e Reforma Agrária. Regras para análise de sementes. Brasília, 1992, 365p. GIULIETTI, A. M.; MENEZES, N. L.; PIRANI, J. R.; MEGURO, M.; WANDERLEY, M. G. L. Flora da Serra do Cipó, Minas Gerais: caracterização e lista das espécies. Boletim de Botânica [Universidade de São Paulo], São Paulo, v9, p. 1-151, 1987.

GIULIETTI, N.; GIULIETTI, A. M.; PIRANI, J.R.; MENEZES, N.L. Estudo em sempre-vivas: importância econômica do extrativismo em Minas Gerais, Brasil. Acta Botânica Brasilica. Brasília, v1, n.2, p.179-193, 1988.

MAGUIRE,J.D. Speed of germination-aid in selection and evaluation for seedling emergence and vigor. Crop Science. Chicago, v.2, n.2, p.176-177, 1962.

MOLDENKE, H.N.; SMITH, L.B. Eriocauláceas. In: Flora

Ilustrada Catarinense. Itajaí: Herbário "Barbosa Rodrigues”, 1976.
NERI, F.C.S.; PAIVA, P.D.O.; BORÉM, R.A.T. Produção e comercialização de sempre-vivas. Informe Agropecuário. Belo Horizonte, v.26, n.227, p.56-61, 2005.

POLLOCK, D.M.; ROSS, E.E. Seed and seedling vigour. In: KOZLOWSKG, T.T. Seed biology. New York: Academic Press, 1972. p.313-387.

ROBERTS, E.H. Viability of seeds. Londres: Chapman and Hall. 1972. 448p.

SCATENA, V.L; LEMOS FILHO,J.P.de; LIMA,A.A.A. Morfologia do desenvolvimento pós-seminal de Syngonathus elegans e $S$. niveus (Eriocaulaceae). Acta Botânica Brasilica. Brasília, v.10, n.1, p.85-91, 1996

TEIXEIRA, A.M. Sempre-vivas: folclore e verdade. Ciência Hoje. Rio de Janeiro, v5, p.14-15, 1987.

YORINORI, J.T. Doenças da soja causadas por fungos. Informe Agropecuário, Belo Horizonte, v.8, n.94, p.40-46, 1982. 